\title{
Adjoint Method and Predictive Control for 1-D Flow in NASA Ames 11-Foot Transonic Wind Tunnel
}

\author{
Nhan Nguyen * \\ NASA Ames Research Center, Moffett Field, CA 94035 \\ Mark Ardema ${ }^{\dagger}$ \\ Santa Clara University, Santa Clara, CA 95053
}

This paper describes a modeling method and a new optimal control approach to investigate a Mach number control problem for the NASA Ames 11-Foot Transonic Wind Tunnel. The flow in the wind tunnel is modeled by the 1-D unsteady Euler equations whose boundary conditions prescribe a controlling action by a compressor. The boundary control inputs to the compressor are in turn controlled by a drive motor system and an inlet guide vane system whose dynamics are modeled by ordinary differential equations. The resulting Euler equations are thus coupled to the ordinary differential equations via the boundary conditions. Optimality conditions are established by an adjoint method and are used to develop a model predictive linear-quadratic optimal control for regulating the Mach number due to a test model disturbance during a continuous pitch.

\section{Introduction}

In this paper, we present a new optimal control approach for a continuous system governed by first order hyperbolic partial differential equations (PDEs) coupled to a discrete system modeled by ordinary differential equations (ODEs) imposed at the continuous system boundary. Hyperbolic equations model many physical systems which are governed by conservation laws such as fluid flow, and therefore have been thoroughly studied. However, problems involving hyperbolic PDEs coupled to ODEs via boundary conditions are much less common. The goal of this paper is to present a model predictive optimal control for solving a Mach number control problem for a wind tunnel using this new modeling approach.

Fig. 1 illustrates the NASA Ames 11-Ft Transonic Wind Tunnel. It is capable of delivering an air speed from Mach 0.2 to Mach 1.5 in a test section measured $11 \mathrm{ft}$ wide by $11 \mathrm{ft}$ high. The air flow is delivered by a compressor driven by a set of synchronous induction AC motors operated in a speed range from 310 to $645 \mathrm{rpm}$ with a total input power of 236,000 horsepowers. The compressor is a variable-geometry machine equipped with inlet guide vanes (IGVs) having adjustable trailing edge flaps through a range of angular deflections from $-7.5^{\circ}$ to $19.5^{\circ}$.

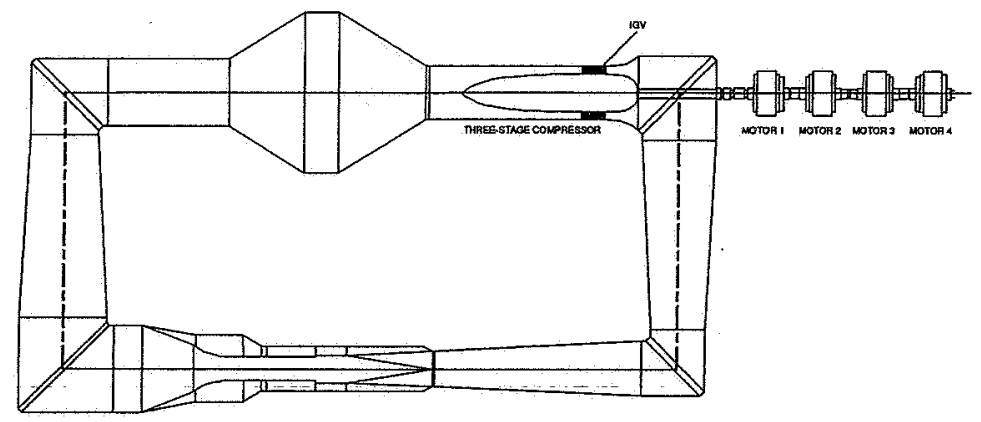

Fig. 1 - NASA Ames 11-Foot Transonic Wind Tunnel

* Research Scientist, Intelligent Systems Division, Mail Stop 269-1, AIAA Member

†Professor, Department of Mechanical Engineering, AIAA Associate Fellow 
The test section aerodynamic condition is normally controlled to ensure that the Mach number variation is within a prescribed tolerance, which can be as small as 0.001 . For subsonic flow, the Mach number control is accomplished by adjusting the IGV flap deflection while holding the compressor speed constant at the discrete set points as shown in Fig. 2 by a compressor speed feedback control.

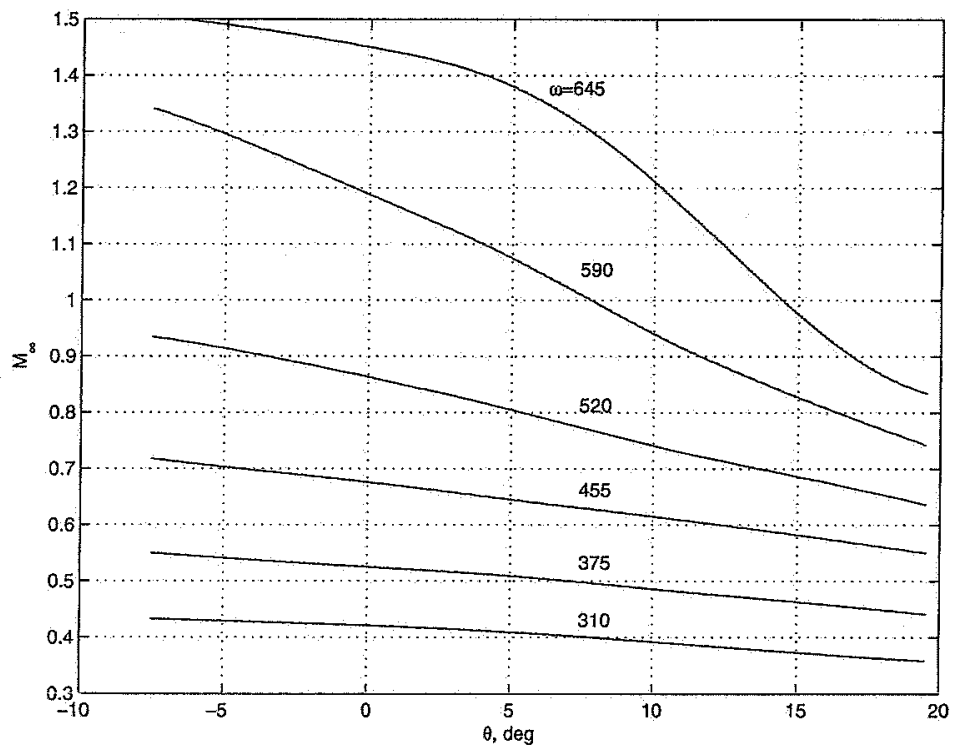

Fig. 2 - NASA Ames 11-Ft Transonic Wind Tunnel Mach Envelope

Typically, during a wind tunnel test, the test model is pitched through a series of angles of attack at a nominal Mach number. This is known as a pitch polar. As the angle of attack increases, the drag force on the test model also increases. This drag force thus translates into a loss in the total pressure across the test model. The total pressure disturbance then propagates downstream to the compressor inlet and sets up a dynamic imbalance in the compressor equilibrium, thereby causing the compressor speed to fall. This consequently results in a deviation in the Mach number in the test section from the desired set point. Presently, to minimize this Mach number deviation, the test model has to be paused in between changes in the pitch angle so that the flow condition can be reestablished by a Mach number feedback control using the IGVs.

It is a recognized that the current Mach number feedback control strategy is deficient and therefore an alternate Mach number control strategy should be considered to provide an effective Mach number control strategy that will allow the test model to be pitched continuously. This strategy would translate into a significant advantage over the current pitch-pause mode. Therefore, the motivation of this study is to investigate a model predictive Mach number optimal control for a wind tunnel based on a modeling approach of coupled hyperbolic PDEs and ODEs.

\section{Wind Tunnel Modeling}

Wind tunnel flow modeling for control design purposes using unsteady fluid dynamics equations has rarely been considered because of the inherent mathematical complexity. Understandably, many wind tunnel control models have been based on heuristic or ad hoc methods. Soeterboek describes a method for control modeling of a test section Mach number by an experimental transfer function coupled with a time delay ${ }^{1}$. The current Mach number control for NASA Ames 11-Ft Transonic Wind Tunnel was developed using a quasi-steady state model based on an empirical relationship between the Mach number and the compressor performance characteristics ${ }^{2}$. The quasi-steady state model circumvents the need for modeling the complex unsteady air flow, but does not capture the time delay effect. As a result, it can lead to poor control handling of disturbance rejection due to the fluid transport delay that exists between the test section and the compressor in a typical wind tunnel.

In this paper, we propose to establish a model of the wind tunnel flow based on the governing conservation laws of physics to capture the fluid transiency. In the process, this model should be more accurate than a heuristic model and 
thus can be used for the proposed predictive Mach number optimal control strategy. The governing Euler equations for an unsteady 1-D flow are ${ }^{3}$

$$
\begin{gathered}
\frac{\partial}{\partial t}(\rho A)+\frac{\partial}{\partial x}(\rho u A)=0 \\
\frac{\partial}{\partial t}(\rho u A)+\frac{\partial}{\partial x}\left(p A+\rho u^{2} A\right)-p \frac{d A}{d x}+\frac{1}{2} \rho u^{2} A \frac{f}{D}=0 \\
\frac{\partial}{\partial t}\left(\rho e A+\frac{1}{2} \rho u^{2} A\right)+\frac{\partial}{\partial x}\left(\rho u e A+\frac{1}{2} \rho u^{3} A+p u A\right)=0
\end{gathered}
$$

where $\rho$ is the static density, $u$ is the flow speed, $p$ is the static pressure, $e$ is the internal energy, $A$ is the cross-sectional area, $D$ is the hydraulic diameter, and $f$ is the friction factor.

Equations (1)-(3) are the conservation equations for fluid flow. It is, however, usually more convenient to express these equations in terms of the mass flow $\dot{m}$, the total pressure $p_{0}$, and the total temperature $T_{0}$. The Mach number can be related to these three quantities by

$$
\dot{m}=\sqrt{\frac{k}{R T_{0}}} p_{0} A M\left(1+\frac{k-1}{2} M^{2}\right)^{-\frac{k+1}{2(k-1)}}
$$

where $M$ is the Mach number and $k$ is the specific heat ratio.

We now introduce an alternate form of the Euler equations as follows

$$
\mathbf{y}_{t}+\mathbf{A}(\mathbf{y}, x) \mathbf{y}_{x}+\mathbf{B}(\mathbf{y}, x)=\mathbf{0}
$$

where $\mathrm{y}(x, t)=\left[\begin{array}{lll}\dot{m} & p_{0} & T_{0}\end{array}\right]^{T}$ is a flow vector and

$$
\mathbf{A}=\left[\begin{array}{ccc}
u & \frac{\dot{p A}}{p_{0}} & \frac{\dot{m} u}{2 T_{0}} \\
\frac{\rho c^{2}}{\rho A} & u\left[1-\frac{(k-1) T}{T_{0}}\right] & \frac{\rho 0 c^{2} u}{T_{0}} \\
\frac{(k-1) T}{\rho A} & -\frac{(k-1)^{2} T u}{k p_{0}} & u\left[1+\frac{(k-1) T}{T_{0}}\right]
\end{array}\right], \mathbf{B}=\left[\begin{array}{c}
\frac{\dot{m} u f}{2 D} \\
\frac{k p_{0} u^{3} f}{2 c^{2} D}\left[1-\frac{(k-1) T}{T_{0}}\right] \\
-\frac{(k-1)^{2} T u^{3} f}{2 c^{2} D}
\end{array}\right]
$$

where $c$ is the speed of sound, $T$ is the static temperature, and $\rho_{0}$ is the total density.

The flow recirculation in a wind tunnel is controlled by a compressor, which creates a pressure rise to compensate for the pressure losses throughout the wind tunnel circuit. We let $x=0$ and $x=L$ be the compressor exit and inlet stations, respectively. Then the boundary conditions for Eq. (5) are specified by the following compressor performance model, which can be obtained empirically from experimental data as

$$
\begin{gathered}
\dot{m}(0, t)=\dot{m}(L, t) \\
p_{0}(0, t)=\left[1+\sum_{i=2}^{4} \sum_{j=0}^{2} \frac{r_{i j} \theta^{j} \omega^{i}}{\sqrt{T_{0}^{i}(L, t)}}\right]\left[b_{1} p_{0}(L, t)-\frac{b_{2} \dot{m}(L, t) \sqrt{T_{0}(L, t)}}{\sum_{i=1}^{3} \sum_{j=0}^{2} \frac{d_{i j} \theta^{j} \omega^{i}}{\sqrt{T_{0}^{i}(L, t)}}}\right] \\
T_{0}(0, t)=T_{0}(L, t)+\frac{b_{2} \omega}{\dot{m}(L, t)}\left[p_{0}(0, t)-p_{0}(L, t)\right]
\end{gathered}
$$

where $b_{i}, d_{i j}, r_{i j}$ are the empirical coefficients.

Equations (6)-(8) are the respective continuity, momentum, and energy equations that relate the mass flow, the total pressure rise, and the temperature rise across the compressor to the compressor speed $\omega$ and the IGV flap deflection $\theta$ which are the control inputs to the compressor. These equations can be written generally as

$$
\mathbf{y}(0, t)=\mathbf{g}(\mathbf{y}(L, t), \mathbf{u})
$$

where $u=\left[\begin{array}{ll}\theta & \omega\end{array}\right]^{T}$ is called a boundary control vector.

The wind tunnel usually operates from one steady state condition to another. Therefore, the initial condition for Eq. (5) is specified as follows

$$
\mathrm{y}(x, 0)=\mathrm{h}(x)
$$


where $\mathbf{h}(x)$ is a steady state solution of Eq. (5).

Since the compressor speed must be controlled simultaneously with the Mach number, we must take into account the dynamics of the drive motors and the IGV system that actually control the compressor speed and IGV flap deflection. The three-phase synchronous induction AC drive motors use a liquid rheostat system as a primary means of changing the rotor resistance $R_{r}$ which changes the motor torque to control the compressor speed. The dynamics of this drive motor system is described by the following motor torque equation

$$
\dot{\omega}=\frac{K_{m} R_{r} \omega_{s}\left(\omega_{s}-\omega\right)}{\left[R_{s}\left(\omega_{s}-\omega\right)+R_{r} \omega_{s}\right]^{2}+L_{s}^{2} \omega_{s}^{2}\left(\omega_{s}-\omega\right)^{2}}-K_{a}\left[p_{0}(0, t)-p_{0}(L, t)\right]
$$

where $R_{s}$ is the stator resistance, $L_{s}$ is the stator inductance, $\omega_{s}$ is the synchronous speed, and $K_{m}$ and $K_{a}$ are the torque constants.

The IGVs are driven by DC servo motors and controlled by the motor field voltage $V_{a}$. The dynamics of this system is modeled as

$$
\dot{\theta}=K_{v} V_{a}-\frac{1}{2} \rho(L, t) u^{2}(L, t)\left(K_{\theta} \theta+K_{c}\right)
$$

where $K_{v}, K_{\theta}, K_{c}$ are the IGV system constants.

We observe that the boundary control inputs $\omega$ and $\theta$ to the compressor become the states or outputs of the drive motors and the IGV system. Both Eqs. (11) and (12) are essentially the actuator dynamics of the compressor and are observed to also depend on the flow variables at the boundary. Thus, these equations are coupled with Eq. (5) through the boundary conditions (6) to (8) and can be expressed in a general form as

$$
\dot{\mathbf{u}}=\mathbf{f}(\mathbf{y}(0, t), \mathbf{y}(\underline{L}, t), \underline{\mathbf{u}}, \mathbf{v})
$$

where $\mathrm{v}=\left[\begin{array}{ll}V_{a} & R_{r}\end{array}\right]^{T}$ is a control vector.

For a well-posed problem, the initial condition for Eq. (13) must be consistent with the initial condition (10) and the boundary condition (9) such that

$$
\mathbf{h}(0)=\mathbf{g}(\mathbf{h}(L), \mathbf{u}(0))
$$

\section{Computational Method}

It can be shown that the eigenvalues of the matrix $\mathbf{A}$ are $\lambda(\mathbf{A})=u, u \pm c$, which are the wave propagation speeds in a fluid medium. Subsonic flow therefore involves two waves propagating downstream and one wave propagating upstream from the source. If $\mathbf{\Lambda}^{+}=\operatorname{diag}(u+c, u, 0)$ is a diagonal matrix of the positive eigenvalues, $\Lambda^{-}=\operatorname{diag}(0,0, u-c)$ is a diagonal matrix of the negative eigenvalues, and $\mathbf{\Phi}$ is a matrix of the right eigenvectors of the matrix $\mathbf{A}$, then the matrix $\mathbf{A}$ can be split into a semi-positive definite matrix and a semi-negative definite matrix as follows

$$
\mathbf{A}=\mathbf{A}^{+}+\mathbf{A}^{-}
$$

where

$$
\begin{aligned}
& \mathbf{A}^{+}=\boldsymbol{\Phi} \Lambda^{+} \boldsymbol{\Phi}^{-1} \\
& \mathbf{A}^{-}=\boldsymbol{\Phi} \Lambda^{-} \Phi^{-1}
\end{aligned}
$$

Equation (5) can be cast into a discretized characteristic form using a combined first order upwind and downwind explicit finite-difference method ${ }^{4}$ as follows

$$
\boldsymbol{\Phi}_{i j}^{-1} \mathbf{y}_{i, j+1}=\mathbf{y}_{i j}-\frac{\Lambda_{i j}^{+} \boldsymbol{\Phi}_{i j}^{-1} \Delta t}{\Delta x}\left(\mathbf{y}_{i j}-\mathbf{y}_{i-1, j}\right)-\frac{\Lambda_{i j}^{-} \boldsymbol{\Phi}_{i j}^{-1} \Delta t}{\Delta x}\left(\mathbf{y}_{i+1, j}-\mathbf{y}_{i j}\right)-\Delta t \Phi_{i j}^{-1} \mathbf{B}_{i j}
$$

where $i=2,3, \ldots, m-1$ denotes the interior points not on the boundary, $j=2,3, \ldots, n-1$ denotes the time step, $\Delta x$ and $\Delta t$ are the space and time steps which must satisfy the following CFL condition ${ }^{4}$

$$
\Delta t<\frac{\Delta x}{u_{\max }+c_{\max }}
$$


For subsonic flow, we require the number of boundary conditions at $x=0$ be equal to the number of positive eigenvalues, or two. We then combine the two boundary conditions (7) and (8) with the characteristic equation in the third row of Eq. (16) to compute the flow variables at $x=0$ as follows

$$
\begin{aligned}
& {\left[\begin{array}{c}
\mathbf{I}_{2} \\
\mathbf{I}_{3} \\
\left(\mathbf{\Psi}_{1 j}\right)_{3}
\end{array}\right] \mathbf{y}_{1, j+1}-\left[\begin{array}{c}
g_{2}\left(\mathbf{y}_{m, j+1}, \mathbf{u}_{j+1}\right) \\
g_{3}\left(\mathbf{y}_{m, j+1}, \mathbf{u}_{j+1}\right) \\
\mathbf{0}_{1 \times 3}
\end{array}\right]=\left[\begin{array}{c}
\mathbf{0}_{1 \times 3} \\
\mathbf{0}_{1 \times 3} \\
\left(\mathbf{\Psi}_{1 j}\right)_{3}
\end{array}\right] \mathbf{y}_{1 j} } \\
&-\frac{\boldsymbol{\Lambda}_{1 j}^{-} \Delta t}{\Delta x}\left[\begin{array}{c}
\mathbf{0}_{1 \times 3} \\
\mathbf{0}_{1 \times 3} \\
\left(\mathbf{\Psi}_{1 j}\right)_{3}
\end{array}\right]\left(\mathbf{y}_{2 j}-\mathbf{y}_{1 j}\right)-\left[\begin{array}{c}
\mathbf{0}_{1 \times 3} \\
\mathbf{0}_{1 \times 3} \\
\left(\mathbf{\Psi}_{1 j}\right)_{3}
\end{array}\right] \Delta t \mathbf{B}_{1 j}
\end{aligned}
$$

where $I_{i}$ is the $i$-th row of a 3-by-3 identity matrix, $0_{1 \times 3}$ is a 1-by-3 zero vector, and $\mathbf{\Psi}=\mathbf{\Phi}^{-1}$.

At $x=L$, we combine the remaining boundary condition (6) with the two remaining characteristic equations from Eq. (16) as follows

$$
\begin{aligned}
{\left[\begin{array}{c}
\left(\mathbf{\Psi}_{m j}\right)_{1} \\
\left(\mathbf{\Psi}_{m j}\right)_{2} \\
\mathbf{I}_{1}
\end{array}\right] \mathbf{y}_{m, j+1}-\left[\begin{array}{c}
\mathbf{0}_{1 \times 3} \\
\mathbf{0}_{1 \times 3} \\
\mathbf{I}_{1}
\end{array}\right] \mathbf{y}_{1, j+1}=\left[\begin{array}{c}
\left(\mathbf{\Psi}_{m j}\right)_{1} \\
\left(\mathbf{\Psi}_{m j}\right)_{2} \\
\mathbf{0}_{1 \times 3}
\end{array}\right] \mathbf{y}_{m j} } \\
-\frac{\mathbf{\Lambda}_{m j}^{+} \Delta t}{\Delta x}\left[\begin{array}{c}
\left(\boldsymbol{\Psi}_{m j}\right)_{1} \\
\left(\mathbf{\Psi}_{m j}\right)_{2} \\
0_{1 \times 3}
\end{array}\right]\left(\mathbf{y}_{m j}-\mathbf{y}_{m-1, j}\right)-\left[\begin{array}{c}
\left(\boldsymbol{\Psi}_{m j}\right)_{1} \\
\left(\mathbf{\Psi}_{m j}\right)_{2} \\
0_{1 \times 3}
\end{array}\right] \Delta t \mathbf{B}_{m j}
\end{aligned}
$$

Both Eqs. (18) and (19) are nonlinear and are coupled together. An iterative method is used to solve for the flow conditions at the compressor inlet and exit simultaneously. Once the solution at the boundary is established, the solution for the interior points can be computed in a time marching procedure.

\section{Validation}

To validate the wind tunnel distributed model, we compute a transition from Mach 0.6 at 455 rpm to Mach 0.9 at $590 \mathrm{rpm}$. The wind tunnel distributed model is discretized into 45 nodes as shown in Fig. 3.

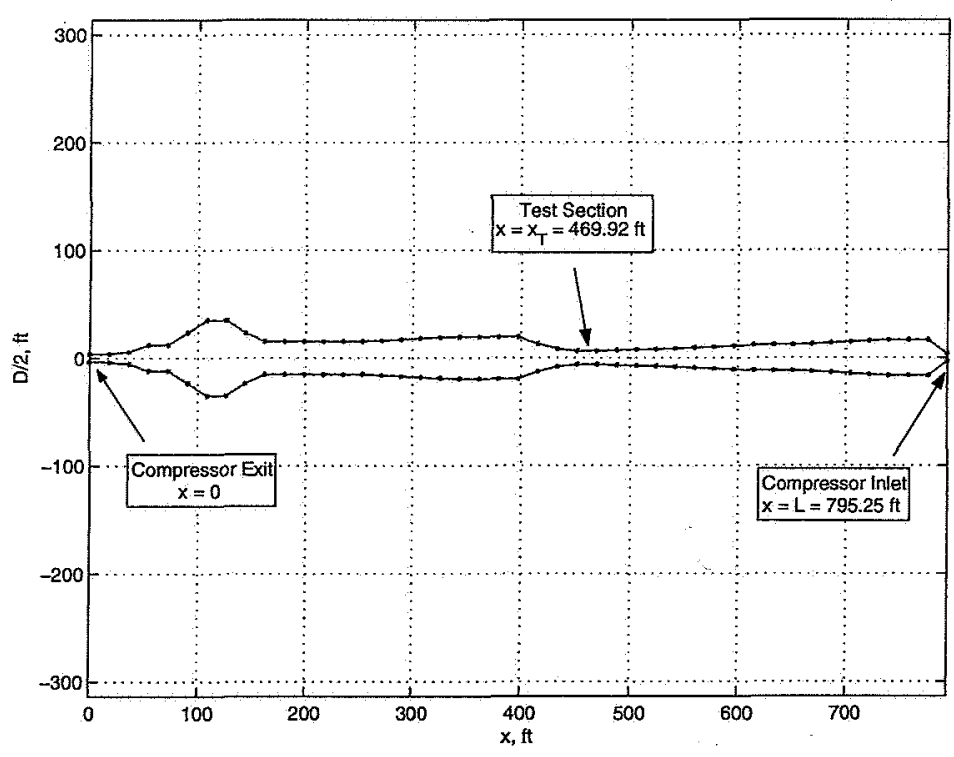

Fig. 3 - Wind Tunnel Discretization

The step size in $x$ is chosen so that important features of the wind tunnel such as the aftercooler and the test section dimensions are captured in the discretization. This step size is found to be $\Delta x=18.07 \mathrm{ft}$. The time step is then 
chosen to be $\Delta t=0.01 \mathrm{sec}$ in order to satisfy the CFL condition (17). At each node, there are three flow quantities, so the discretized model contains a total of 135 aerodynamic states plus two compressor dynamic states and two control inputs.

The time histories of the compressor speed and the IGV flap deflection during the Mach number transition are shown in Fig. 4. The IGV flap deflection is first adjusted to its maximum value while the compressor speed is regulated at $455 \mathrm{rpm}$. A new compressor speed set point command at $590 \mathrm{rpm}$ is then sought while the IGV flap deflection is maintained at its maximum value. When the compressor settles to the new set point, the IGV flaps are then actuated until the Mach number reaches 0.9. A small ripple in the compressor speed occurs at the beginning of the final IGV flap actuation due to an integral feedback control regulating the motor speed set point as a result of the changing aerodynamic torque during the IGV actuation.

Fig. 5 shows the resulting Mach number variation throughout the transition. The computed Mach number from the distributed model agrees very well with the experimental data, thus validating the modeling approach.

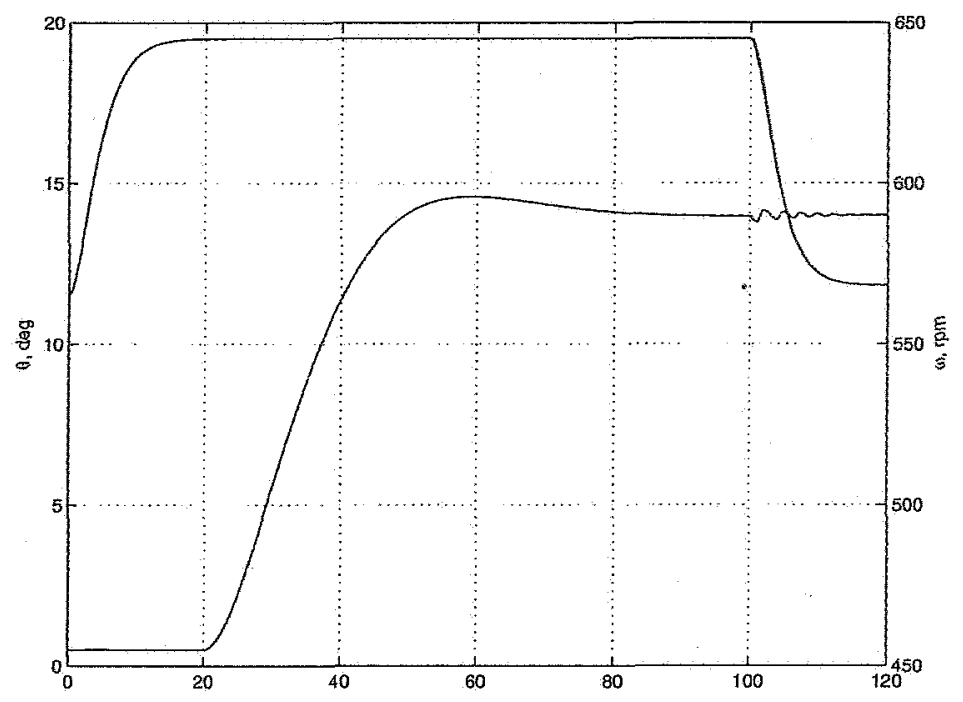

Fig. 4 - Compressor Control Time History

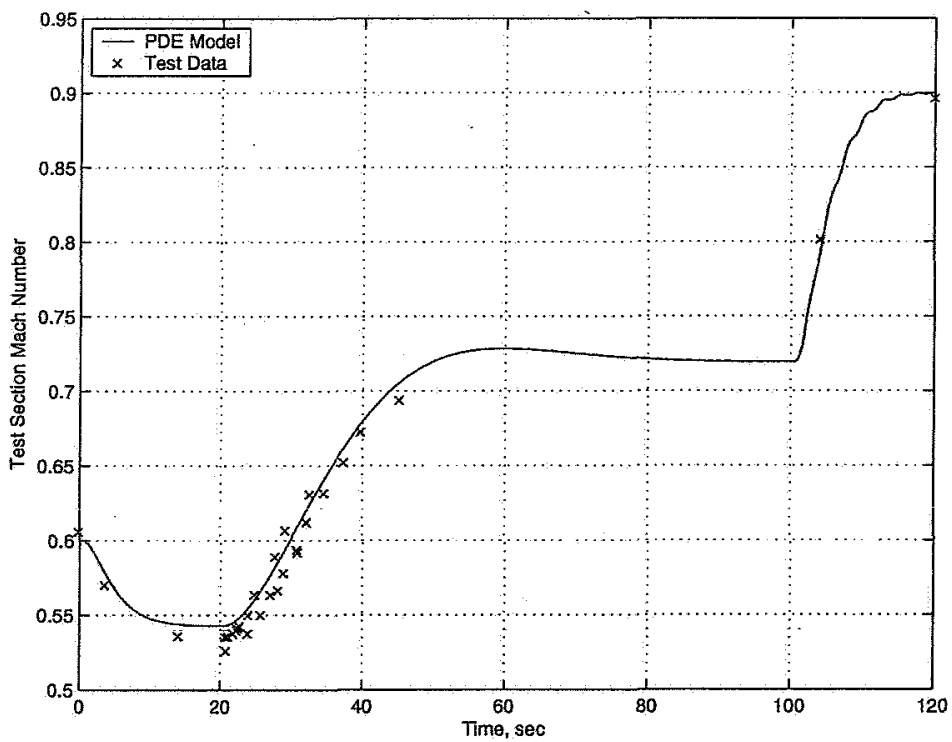

Fig. 5 - Mach Number Response

American Institute of Aeronautics and Astronautics 
Fig. 6 is the plot of the Mach number distribution along the wind tunnel showing the Mach number response in the test section. The demonstrated accuracy of the wind tunnel model is critical to the model predictive Mach number control strategy wherein the control inputs are strictly computed from the mathematical model of the wind tunnel.

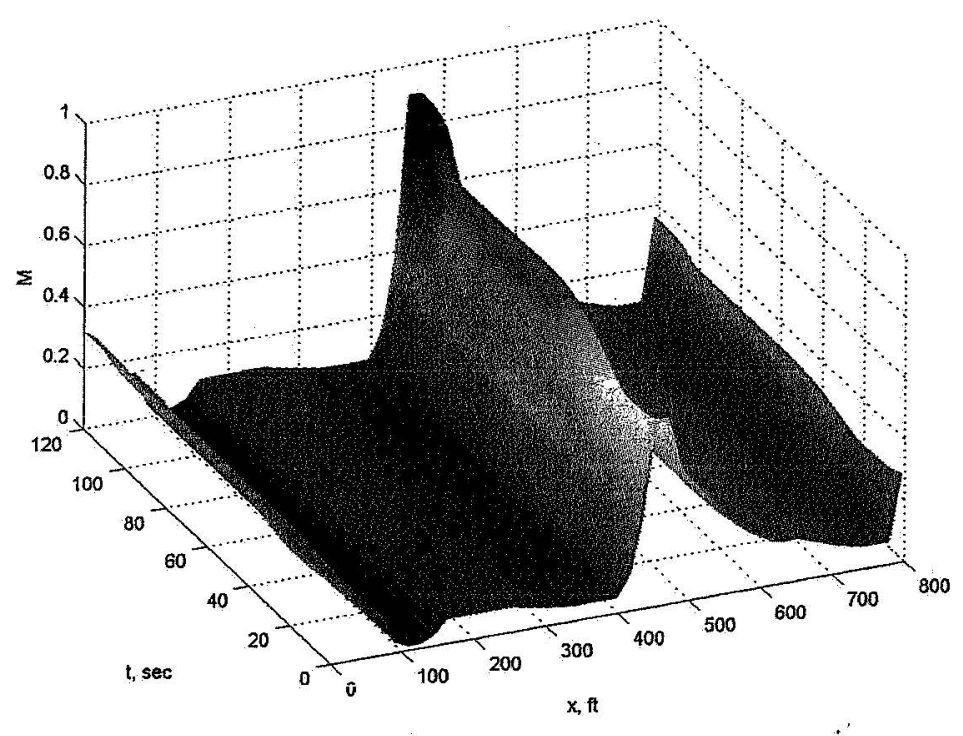

Fig. 6 - Mach Number Distribution

\section{Adjoint Method}

In order to develop a model predictive Mach number optimal control, we propose to establish an optimal control theory for systems modeled by PDEs coupled to ODEs via boundary conditions based on an adjoint method ${ }^{5}$. Optimal control for PDE systems has been examined in the literature ${ }^{6,7,8}$, but little existing theory has been found for the systems of the present interest. The adjoint method has been used in many fluid optimization problems ${ }^{9,10}$. There are two approaches to the adjoint formulation in the optimization application domain. This first approach is to formulate the adjoint method directly to the fluid equations of motion. This is called a continuous adjoint method ${ }^{11}$. The other approach is to formulate the adjoint method to the discretized equations of motion and as such is called a discrete adjoint method. Studies have found that the continuous adjoint method is generally preferred ${ }^{11}$. We adopt the continuous adjoint approach in this study. Towards that end, we are interested in obtaining an optimal control that will minimize the responses at the system boundaries and the boundary control action by considering the following linear-quadratic cost function with a fixed terminal time $t_{f}$

$$
\min J(\mathbf{y}(0, t), \mathbf{y}(L, t), \mathbf{u}, \mathbf{v})=\frac{1}{2} \int_{0}^{t_{f}}\left[\mathbf{y}^{T}(0, t) \mathbf{P y}(0, t)+\mathbf{y}^{T}(L, t) \mathbf{S y}(L, t)+\mathbf{u}^{T} \mathbf{Q u}+\mathbf{v}^{T} \mathbf{R v}\right] d t
$$

subject to Eq. (5) coupled to Eq. (12) via the boundary condition (9), where $P, S$ and $Q$ are semi-positive definite symmetric matrices, and $\mathbf{R}$ is a positive definite symmetric matrix.

We define $\lambda(x, t)$ as the adjoint vector for Eq. (5) and $\mu(t)$ as the adjoint vector for Eq. (12). Using the Lagrange multiplier method, the augmented cost function becomes

$$
\begin{aligned}
J(\mathbf{y}(0, t), \mathbf{y}(L, t), \mathbf{u}, \mathbf{v})=-\int_{0}^{t_{f}} \int_{0}^{L} \lambda^{T}\left(\mathbf{y}_{t}+\mathbf{A} \mathbf{y}_{x}+\mathbf{B}\right) d x d t \\
+\int_{0}^{t_{f}}\left[\frac{1}{2} \mathbf{y}^{T}(0, t) \mathbf{P y}(0, t)+\frac{1}{2} \mathbf{y}^{T}(L, t) \mathbf{S y}(L, t)+\frac{1}{2} \mathbf{u}^{T} \mathbf{Q u}+\frac{1}{2} \mathbf{v}^{T} \mathbf{R v}+\mu(-\dot{\mathbf{u}}+\mathbf{f})\right] d t
\end{aligned}
$$


To establish the necessary conditions of optimality, we compute the first variation of the augmented cost function using the Green's theorem which results in

$$
\begin{aligned}
& \delta J=\int_{0}^{t_{f}} \int_{0}^{L} \delta \mathbf{y}^{T}\left[\lambda_{t}+\left(\mathbf{A}^{T} \lambda\right)_{x}-\mathbf{B}_{\mathbf{y}}^{T} \lambda\right]^{T} d x d t+\int_{0}^{L} \delta \mathbf{y}^{T}\left(x, t_{f}\right) \boldsymbol{\lambda}\left(x, t_{f}\right) d x \\
&-\int_{0}^{t_{f}}\left[\delta \mathbf{y}^{T}(L, t) \mathbf{A}^{T}(\mathbf{y}(L, t), L) \boldsymbol{\lambda}(L, t)-\delta \mathbf{y}^{T}(0, t) \mathbf{A}^{T}(\mathbf{y}(0, t), 0) \boldsymbol{\lambda}(0, t)\right] d t \\
&+\int_{0}^{t_{f}}\left\{\delta \mathbf{y}^{T}(0, t)[\right.\left.\mathbf{P y}(0, t)+\mathbf{f}_{\mathbf{y}(0, t)}^{T} \boldsymbol{\mu}\right]+\delta \mathbf{y}^{T}(L, t)\left[\mathbf{S y}(L, t)+\mathbf{f}_{\mathbf{y}(L, t)}^{T} \boldsymbol{\mu}\right] \\
&\left.+\delta \mathbf{u}^{T}\left(\mathbf{Q u}+\mathbf{f}_{\mathbf{u}}^{T} \boldsymbol{\mu}+\dot{\mu}\right)+\delta \mathbf{v}^{T}\left(\mathbf{R v}+\mathbf{f}_{\mathbf{v}}^{T} \boldsymbol{\mu}\right)\right\} d t-\delta \mathbf{u}^{T}\left(t_{f}\right) \boldsymbol{\mu}\left(t_{f}\right)
\end{aligned}
$$

In addition, we also compute the first variation of the boundary condition (9) as follows

$$
\delta \mathbf{y}^{T}(0, t)=\delta \mathbf{y}^{T}(L, t) \mathbf{g}_{\mathbf{y}(L, t)}^{T}+\delta \mathbf{u}^{T} \mathbf{g}_{\mathbf{u}}^{T}
$$

Substituting Eq. (23) into Eq. (22) and setting $\delta J=0$, we obtain the following adjoint PDE

$$
\lambda_{t}+\left(\mathbf{A}^{T} \lambda\right)_{x}-\mathbf{B}_{\mathbf{y}}^{T} \boldsymbol{\lambda}=0
$$

subject to a boundary condition

$\mathbf{A}^{T}(\mathbf{y}(L, t), L) \boldsymbol{\lambda}(L, t)=\mathbf{g}_{\mathbf{y}(L, t)}^{T} \mathbf{A}^{T}(\mathbf{y}(0, t), 0) \boldsymbol{\lambda}(0, t)+\left[\mathbf{f}_{\mathbf{y}(L, t)}^{T}+\mathbf{g}_{\mathbf{y}(L, t)}^{T} \mathbf{f}_{\mathbf{y}(0, t)}^{T}\right] \boldsymbol{\mu}+\mathbf{g}_{\mathbf{y}(L, t)}^{T} \mathbf{P y}(0, t)+\mathbf{S y}(L, t)$

and a terminal time condition

$$
\lambda\left(x, t_{f}\right)=0
$$

In addition, we obtain the following adjoint ODE

$$
\dot{\boldsymbol{\mu}}=-\mathbf{g}_{\mathbf{u}}^{T} \mathbf{A}^{T}(\mathbf{y}(0, t), 0) \boldsymbol{\lambda}(0, t)-\left[\mathbf{f}_{\mathbf{u}}^{T}+\mathbf{g}_{\mathbf{u}}^{T} \mathbf{f}_{\mathbf{y}(0, t)}^{T}\right] \boldsymbol{\mu}-\mathbf{Q u}-\mathbf{g}_{\mathbf{u}}^{T} \mathbf{P y}(0, t)
$$

subject to a terminal time condition

$$
\mu\left(t_{f}\right)=0
$$

Equations (24) to (28) constitute an adjoint system for the original problem defined by Eqs. (5), (9), (10), (12), and (14). These systems represent a duality wherein the adjoint variables are mapped into space and time reversal coordinates. Solutions for such systems are generally complex for nonlinear problems and are usually solved by gradient methods. For linear problems, they can be solved by other methods which will be demonstrated by applying the adjoint method to the Mach number control problem.

\section{Model Predictive Mach Number Optimal Control}

The optimal control results can now be applied to design a predictive Mach number control for a wind tunnel. One of the objectives of a wind tunnel Mach number control is to control the Mach number in the test section due to a small total pressure disturbance created by the test model drag as accurately as possible. Neglecting the continuity and energy equations since the mass flow and total temperature are less sensitive to the total pressure disturbance caused by the test model than the total pressure, then linearizing Eq. (5) about a steady state nominal operating condition results in a linearized momentum equation

$$
\frac{1}{\alpha(x)} \frac{\partial y}{\partial t}+\frac{\partial y}{\partial x}+\beta(x, t) y+w(t)=0
$$

where $y(x, t)=\Delta p_{0}(x, t)$ is the total pressure perturbation and

$$
\alpha(x)=\bar{M}(x) \bar{c}(x) \frac{4-2 k+(k-1) \bar{M}^{2}(x)}{2+(k-1) \bar{M}^{2}(x)}
$$




$$
\begin{gathered}
\beta(x, t)=-\frac{k \bar{M}^{2}(x)\left[1+k \bar{M}^{2}(x)\right]}{2\left[1-\bar{M}^{2}(x)\right]}\left[\frac{f(x)}{D(x)}+\frac{C_{D}(t) A_{m}}{A\left(x_{T}\right) L_{m}}\right] \\
w(t)=\frac{k}{2} \overline{p_{0}}\left(x_{T}\right) \bar{M}^{2}\left(x_{T}\right) \frac{C_{D}(t) A_{m}}{A\left(x_{T}\right) L_{m}}
\end{gathered}
$$

where the overbar denotes the nominal steady state condition and $w(t)$ is the total pressure disturbance as a function of the nominal Mach number $\bar{M}$ in the test section at $x=x_{T}$, the test model drag coefficient $C_{D}$, the test model reference area $A_{m}$, and the reference length $L_{m}$. The validity of this equation excludes the transonic region when $\dot{M}\left(x_{T}\right) \simeq 1$.

Linearizing Eq. (7) results in the following boundary condition

$$
y(0, t)=\mathbf{G} \Delta \mathbf{u}(t)+H y(L, t)
$$

where $\Delta \mathbf{u}=\left[\begin{array}{ccc}\Delta \theta & \Delta \omega & \xi\end{array}\right]^{T}$ is a compressor boundary control error vector comprising the IGV flap deflection error $\Delta \theta$, the compressor speed error $\Delta \omega$, and the compressor speed error integral $\xi=\int_{0}^{t} \Delta \omega d \tau$. The error integral is designed to achieve a zero steady state error in the compressor speed. In essence, the control scheme is a proportionalintegral control. The matrix $\mathbf{G}=\left[\begin{array}{lll}\frac{\partial p_{0}(0, t)}{\partial \theta} & \frac{\partial p_{0}(0, t)}{\partial \omega} & 0\end{array}\right]$ and $H=\frac{\partial p_{0}(0, t)}{\partial p_{0}(L, t)}$ are evaluated from Eq. (7).

The compressor boundary control error dynamics is obtained by linearizing Eq. (13)

$$
\Delta \dot{\mathbf{u}}=\mathbf{C} \Delta \mathbf{u}+\mathbf{D} \Delta \mathbf{v}+\mathbf{E} y(0, t)+\mathbf{F} y(L, t)
$$

where $\Delta \mathrm{v}=\left[\begin{array}{cc}\Delta V_{a} & \Delta R_{r}\end{array}\right]^{T}$ is the control perturbation vector and

$$
\mathbf{C}=\left[\begin{array}{ccc}
\frac{\partial \dot{\theta}}{\partial \theta} & \frac{\partial \dot{\theta}}{\partial \dot{\omega}} & 0 \\
\frac{\partial \omega}{\partial \theta} & \frac{\partial \omega}{\partial \omega} & 0 \\
0 & 1 & 0
\end{array}\right], \mathbf{D}=\left[\begin{array}{cc}
\frac{\partial \dot{\theta}}{\partial V} & 0 \\
0 & \frac{\partial \dot{\omega}}{\partial R_{r}} \\
0 & 0
\end{array}\right], \mathbf{E}=\left[\begin{array}{c}
0 \\
\frac{\partial \dot{\omega}}{\partial p_{0}(0, t)} \\
0
\end{array}\right], \mathbf{F}=\left[\begin{array}{c}
\frac{\partial \dot{\theta}}{\partial p_{0}(L, t)} \\
\frac{\partial \dot{\omega}}{\partial p_{0}(L, t)} \\
0
\end{array}\right]
$$

To design a Mach number control, we recognize that the Mach number perturbation is caused by a total pressure disturbance downstream of the test model. Since there is no total pressure disturbance upstream of the test model, to minimize the Mach number perturbation, we would need to minimize the total pressure perturbation at the compressor exit at $x=0$. Thus, we consider the following linear-quadratic cost function

$$
J=\int_{0}^{t_{f}}\left[\frac{1}{2} P y^{2}(0, t)+\frac{1}{2} \Delta \mathbf{u}^{T} \mathbf{Q} \Delta \mathbf{u}+\frac{1}{2} \Delta \mathbf{v}^{T} \mathbf{R} \Delta \mathbf{v}\right] d t
$$

where $P \geq 0, \mathbf{Q} \geq \mathbf{0}$, and $\mathbf{R}>\mathbf{0}$.

Applying the necessary conditions of optimality results in the following adjoint equations

$$
\begin{gathered}
\frac{\partial \lambda}{\partial t}+\frac{\partial}{\partial x}(\alpha \lambda)-\beta \alpha \lambda=0 \\
\alpha(L) \lambda(L, t)=H \alpha(0) \lambda(0, t)+H P y(0, t)+\left(\mathbf{F}^{T}+H \mathbf{E}^{T}\right) \boldsymbol{\mu} \\
\dot{\boldsymbol{\mu}}=-\mathbf{Q} \Delta \mathbf{u}-\left(\mathbf{C}^{T}+\mathbf{G}^{T} \mathbf{E}^{T}\right) \boldsymbol{\mu}-\mathbf{G}^{T} \alpha(0) \lambda(0, t)-\mathbf{G}^{T} P y(0, t) \\
\mathbf{R} \Delta \mathbf{v}+\mathbf{D}^{T} \boldsymbol{\mu}=0
\end{gathered}
$$

It can be shown that the general solution of Eq. (29) is

$$
y(x, t)=a(x, t)\left[f\left(t-t_{d}(x)\right)-q(x, t)\right]
$$

where

$$
\begin{gathered}
t_{d}(x)=\int_{0}^{x} \frac{d \sigma}{\alpha(\sigma)} \\
a(x, t)=e^{-\int_{0}^{x} \beta\left(\sigma, t-t_{d}(x)+t_{d}(\sigma)\right) d \sigma}
\end{gathered}
$$




$$
q(x, t)=\int_{0}^{x} a^{-1}\left(\sigma, t-t_{d}(x)+t_{d}(\sigma)\right) w\left(t-t_{d}(x)+t_{d}(\sigma)\right) d \sigma
$$

We now consider two special cases: a short time horizon when $t_{f}<t_{L}$, where $t_{L}=t_{d}(L)$ is a fluid transport delay, and a long time horizon when $t_{f} \rightarrow \infty$. For a short time horizon, we have the following relationship

$$
\begin{gathered}
y(0, t)=\mathbf{G} \Delta \mathbf{u}(t)-H a(L, t) q(L, t) \\
y(L, t)=-a(L, t) q(L, t) \\
\alpha(0) \lambda(0, t)=0
\end{gathered}
$$

On the other hand, for a long time horizon, we obtain the following quasi-steady state solution

$$
\begin{gathered}
y(0, t)=[1-H a(L, t)]^{-1}[\mathbf{G} \Delta \mathbf{u}(t)-H a(L, t) q(L, t)] \\
y(L, t)=a(L, t)[1-H a(L, t)]^{-1}[\mathbf{G} \Delta \mathbf{u}(t)-H a(L, t) q(L, t)]-a(L, t) q(L, t) \\
\alpha(0) \lambda(0, t)=a(L, t)[1-H a(L, t)]^{-1}\left[H P y(0, t)+\left(\mathbf{F}^{T}+H \mathbf{E}^{T}\right) \mu(t)\right]
\end{gathered}
$$

We now define a time horizon parameter $\gamma$ which takes on a value ranging from 0 to 1 that represents the effect of the time horizon on the optimal control solution such that $\gamma=0$ corresponds to a short time horizon and $\gamma=1$ corresponds to a long time horizon. The optimal control solution can now be obtained from the following the Riccati equation by letting $\mu=\mathrm{W} \Delta \mathbf{u}+\mathrm{V}$

$$
\begin{gathered}
\dot{\mathbf{W}}+\mathrm{WC}_{e}+\mathrm{C}_{e}^{T} \mathbf{W}-\mathrm{WDR}^{-1} \mathrm{D}^{T} \mathbf{W}+\mathrm{Q}_{e}=\mathbf{0} \\
\dot{\mathbf{V}}+\mathrm{C}_{\mathrm{e}}{ }^{T} \mathrm{~V}-\mathrm{WDR}^{-1} \mathbf{D}^{T} \mathbf{V}-\mathbf{W} \mathbf{S}-\mathbf{U}=\mathbf{0}
\end{gathered}
$$

where

$$
\begin{gathered}
\mathbf{C}_{e}=\mathbf{C}+\mathbf{E G}+\gamma(\mathbf{F}+\mathbf{E} H) \mathbf{G} a(L, t)[1-\gamma H a(L, t)]^{-1} \\
\mathbf{Q}_{e}=\mathbf{Q}+\mathbf{G}^{T} P \mathbf{G}[1-\gamma H a(L, t)]^{-2} \\
\mathbf{S}=(\mathbf{F}+\mathbf{E} H) a(L, t) q(L, t)[1-\gamma H a(L, t)]^{-1} \\
\mathbf{U}=\mathbf{G}^{T} P H a(L, t) q(L, t)[1-\gamma H a(L, t)]^{-2}
\end{gathered}
$$

The control perturbation $\Delta v$ therefore is expressed as a feedback control as

$$
\Delta \mathbf{v}(t)=-\mathbf{R}^{-1} \mathbf{D}^{T} \mathbf{W}(t) \Delta \mathbf{u}(t)-\mathbf{R}^{-1} \mathbf{D}^{T} \mathbf{V}(t)
$$

Equation (46) compute the corrective control inputs for the drive motors and the IGV system which are to be added to the steady state control inputs at a nominal Mach number to give the total control inputs for the drive motors and IGV system according to

$$
\left[\begin{array}{l}
V_{a}(t) \\
R_{r}(t)
\end{array}\right]=\left[\begin{array}{c}
\bar{V}_{a} \\
\bar{R}_{r}
\end{array}\right]+\left[\begin{array}{c}
\Delta V_{a}(t) \\
\Delta R_{r}(t)
\end{array}\right]
$$

However, we recognize that the compressor generally does not sense the effect of the total pressure disturbance signal caused by the motion of the test model at the same time instance as the disturbance itself. Because of the fluid transport delay, there is a time lag between the test section and the compressor inlet such that the compressor dynamic response lags the total pressure disturbance in the test section by a time delay $\Delta t_{d}=t_{d}(L)-t_{d}\left(x_{T}\right)$. This lag time generally will cause the Mach number to drop before the drive motor and IGV system control inputs can correct for it. Thus, to account for this time delay, the drive motor and IGV system control inputs must precede the total pressure disturbance in the test section by the same time lag. In effect, this is the essence of the proposed model predictive Mach number control strategy whereby the control inputs have to be computed from an accurate mathematical model of the wind tunnel in a future time prior to the start of a continuous-pitch polar when the error signals are not yet available. In contrast with the current Mach number feedback control strategy, the model predictive Mach number optimal control is a feedforward, open-loop scheme. The overall drive motor and IGV system model predictive control inputs can now be computed as

$$
\left[\begin{array}{l}
V_{a}(t) \\
R_{r}(t)
\end{array}\right]=\left[\begin{array}{c}
\bar{V}_{a} \\
\bar{R}_{r}
\end{array}\right]+\left[\begin{array}{c}
\Delta V_{a}\left(t+\Delta t_{d}\right) \\
\Delta R_{r}\left(t+\Delta t_{d}\right)
\end{array}\right]
$$

10

American Institute of Aeronautics and Astronautics 
To demonstrate the effectiveness of the proposed model predictive Mach number optimal control, we select a test section Mach number $\bar{M}\left(x_{T}\right)=0.6$, a test section total pressure $\overline{p_{0}}\left(x_{T}\right)=2116 \mathrm{psf}$. We conduct a simulation for a test model being pitched continuously from $0^{\circ}$ to $15^{\circ}$ in $30 \mathrm{sec}$ with a total pressure disturbance as shown in Fig. 7.

The compressor speed is nominally at $455 \mathrm{rpm}$ and the IGV flap deflection is at $11.5^{\circ}$. The geometry information is taken from the NASA Ames 11-Foot Transonic Wind Tunnel with a length $L=795 \mathrm{ft}$ and the test section at $x_{T}=470$ $\mathrm{ft}$. The period is computed to be $t_{L}=15.6 \mathrm{sec}$, and the time delay between the test section and the compressor inlet is computed to be $\Delta t_{d}=3.6 \mathrm{sec}$. The system matrices are $\mathbf{G}=\left[\begin{array}{lll}25.5415 & -684.4960 & 0\end{array}\right], H=1.6013$, and

$$
\begin{gathered}
\mathbf{C}=\left[\begin{array}{ccc}
-0.0597 & 1.3223 & 0 \\
0 & -1.3565 \times 10^{-5} & 0 \\
1 & 0 & 0
\end{array}\right], \mathbf{D}=\left[\begin{array}{cc}
-0.3254 & 0 \\
0 & 7.6358 \times 10^{-5} \\
0 & 0
\end{array}\right] \\
\mathbf{E}=\left[\begin{array}{c}
-0.0019 \\
0 \\
0
\end{array}\right], \mathbf{F}=\left[\begin{array}{c}
-0.0012 \\
3.1673 \times 10^{-9} \\
0
\end{array}\right]
\end{gathered}
$$

The weighting matrices are selected to be $P=0.001, \mathbf{Q}=\operatorname{diag}(0,0.01,0.01), \mathbf{R}=\operatorname{diag}\left(1 \times 10^{-6}, 1,0\right)$.

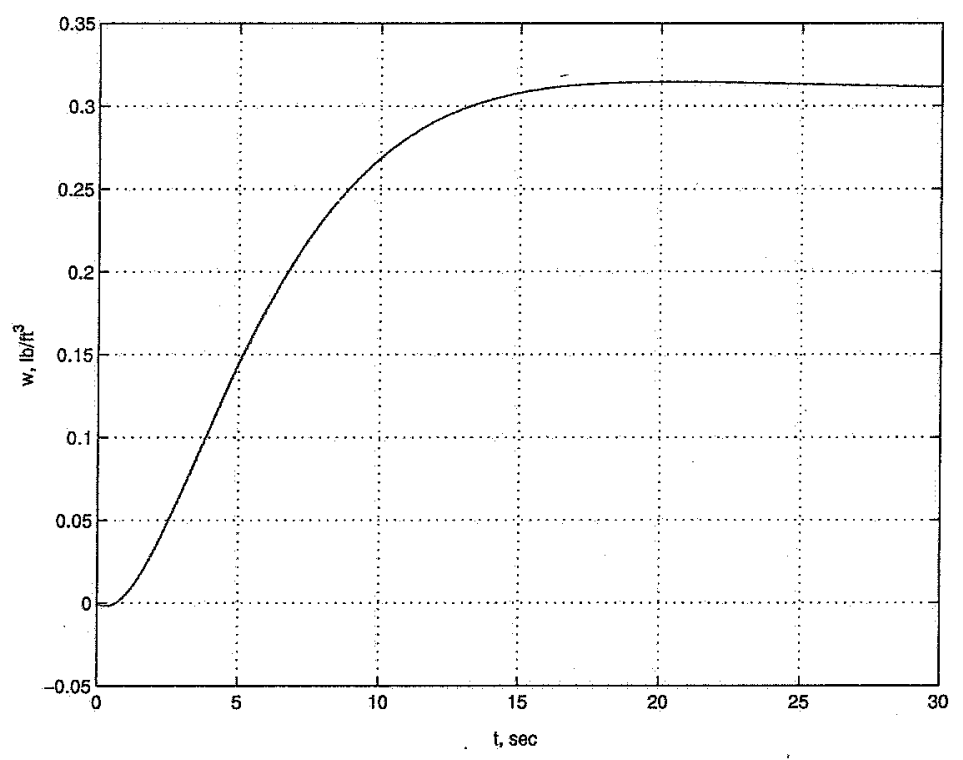

Fig. 7 - Test Section Total Pressure Disturbance

The results of the control simulation are presented in Figs. 8 to 13. Fig. 8 is a plot of the feedback IGV field voltage control perturbation and Fig. 9 is the plot of the drive motor rotor resistance control perturbation for both $\gamma=0$ and $\gamma=1$. The IGV and the drive motor control perturbation inputs for $\gamma=1$ are greater in amplitude than those for $\gamma=0$ as expected since the time horizon parameter $\gamma$ indicates the degree of a control effort which is the largest for an infinite time horizon control. The effect of fluid transport delay in the first 3 sec duration is noted when the control inputs are essentially zero. The model predictive control scheme according to Eq. (49) in effect is designed to remove this time delay in the control inputs.

With reference to Fig. 10, the test section Mach number responses for various control schemes are computed from the fully nonlinear model. The feedback control schemes for both $\gamma=0$ and $\gamma=1$ clearly are not able to maintain the Mach number to within the tolerance band during the disturbance generated by the continuous pitch of the test model in the first $15 \mathrm{sec}$. Because of the time delay during which the feedback control inputs are inactive as seen in Figs. 8 and 9, the Mach number drops below the tolerance band before the error signals become available from the sensors at the compressor to allow the feedback control inputs to be computed. Once the feedback control inputs are applied to the drive motors and the IGV system, the Mach number begins to stabilize and cause the Mach number to eventually return to its desired set point. 


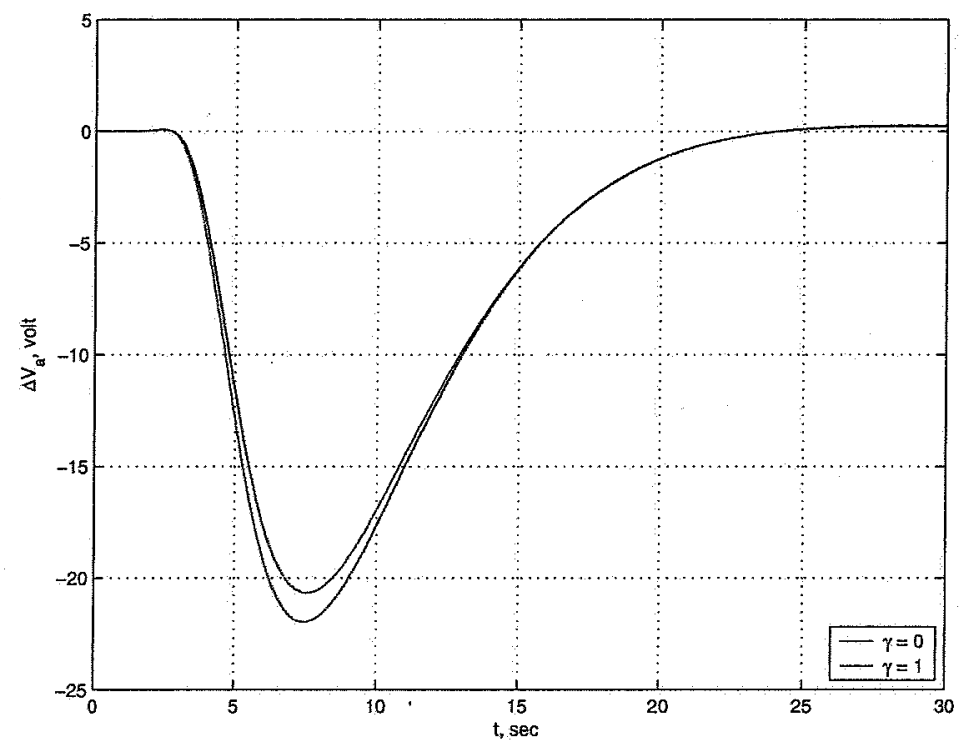

Fig. 8 - IGV Field Voltage Perturbation

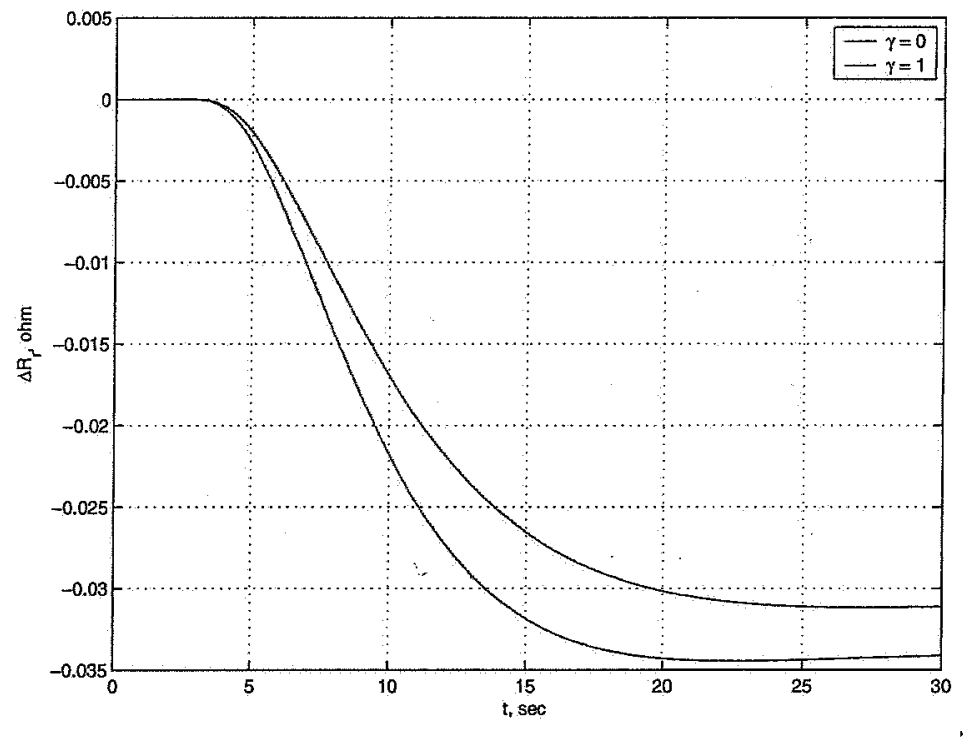

Fig. 9 - Drive Motor Rotor Resistance Perturbation

In contrast, the model predictive control scheme apparently is much more effective in handling the total pressure disturbance. The model predictive control scheme for $\gamma=0$ is an improving control relative to the feedback control schemes since it reduces the deviation in the Mach number to within the tolerance band, but suffers a steady state error. In contrast, the model predictive control scheme for $\gamma=1$ is clearly superior to all other schemes in that it is able to hold the Mach number to well within the tolerance band throughout the time horizon and converges to the set point faster than all other control schemes. This clearly demonstrates the effect of the time horizon parameter $\gamma$. We also note that if thère were no corrective control, the Mäch number would have continued to fall off until it would have reached a new steady state value. 


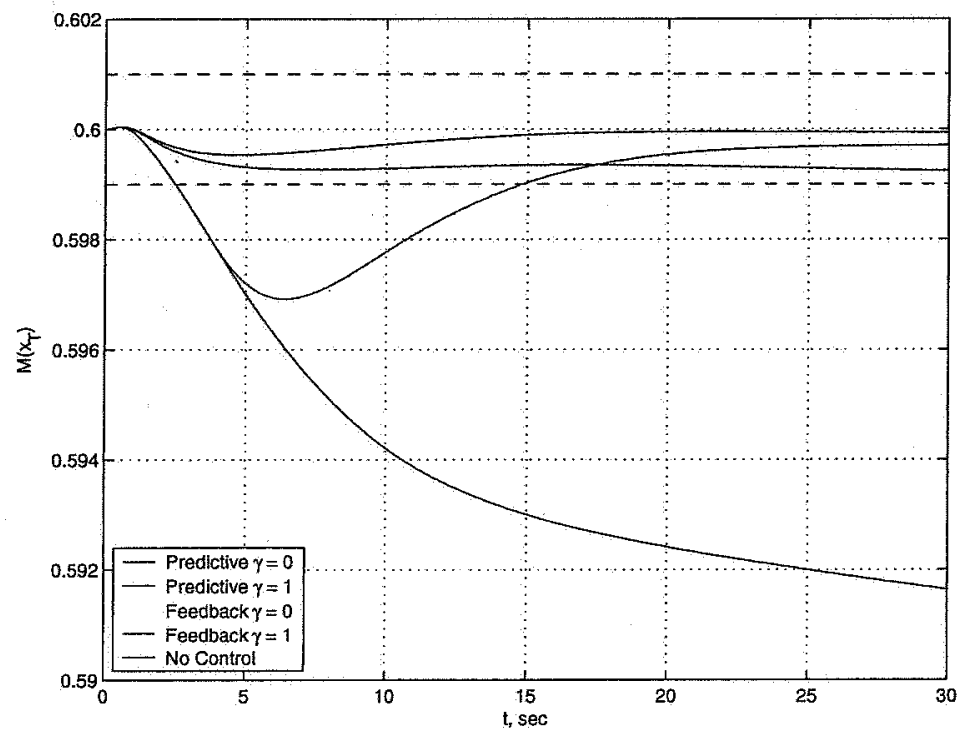

Fig. 10 - Mach Number Response

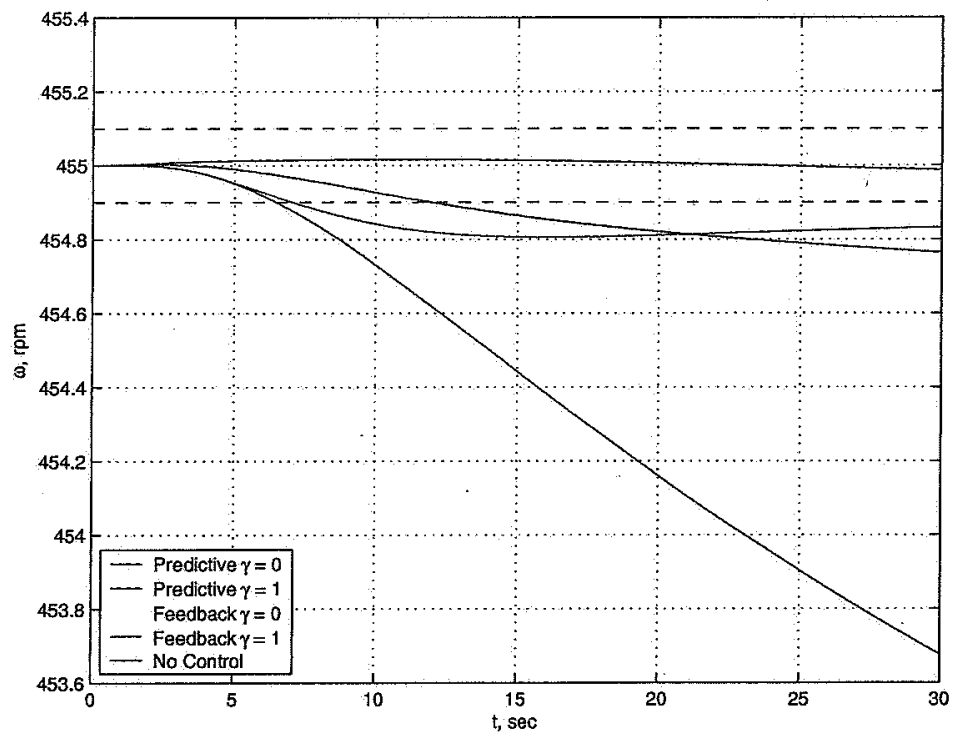

Fig. 11 - Compressor Speed Response

Fig. 11 shows the compressor speed responses to various control schemes. The model predictive control scheme for $\gamma=1$ provides a much better regulation than all other schemes. Due to the integral action, the compressor speed error is virtually right on the set point and well within the desired tolerance of $\pm 0.1 \mathrm{rpm}$. Both the model predictive control scheme and the feedback control scheme for $\gamma=0$ appear to suffer a slightly poor regulation as the compressor speed error does not tend to zero. This is obviously a result of insufficient integral control efforts for a * short time horizon control. It is also noted that if there were no corrective control, the compressor speed would have continued to drop until a new steady state set point would have been established.

The IGV flap deflection responses are presented in Fig. 12. The differences between the model predictive control scheme and the feedback control scheme illustrate the effect of the time delay quite clearly. Since the compressor speed 
is not sensitive to various control schemes, it can be seen that the test section Mach number response is predominantly controlled by the IGV flap deflection.

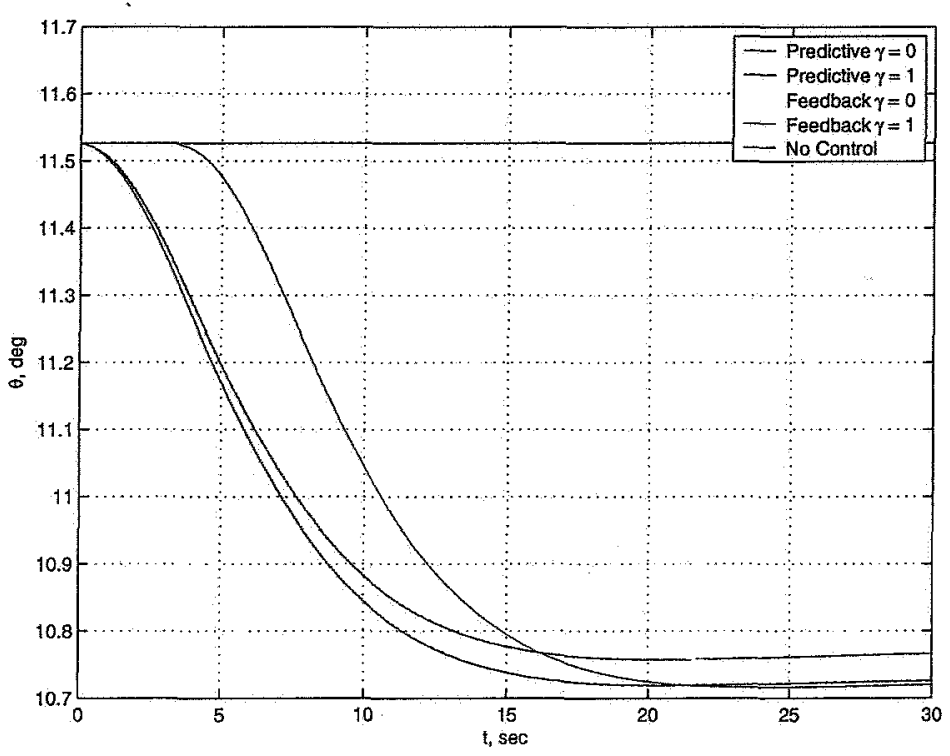

Fig. 12 - IGV flap deflection Response

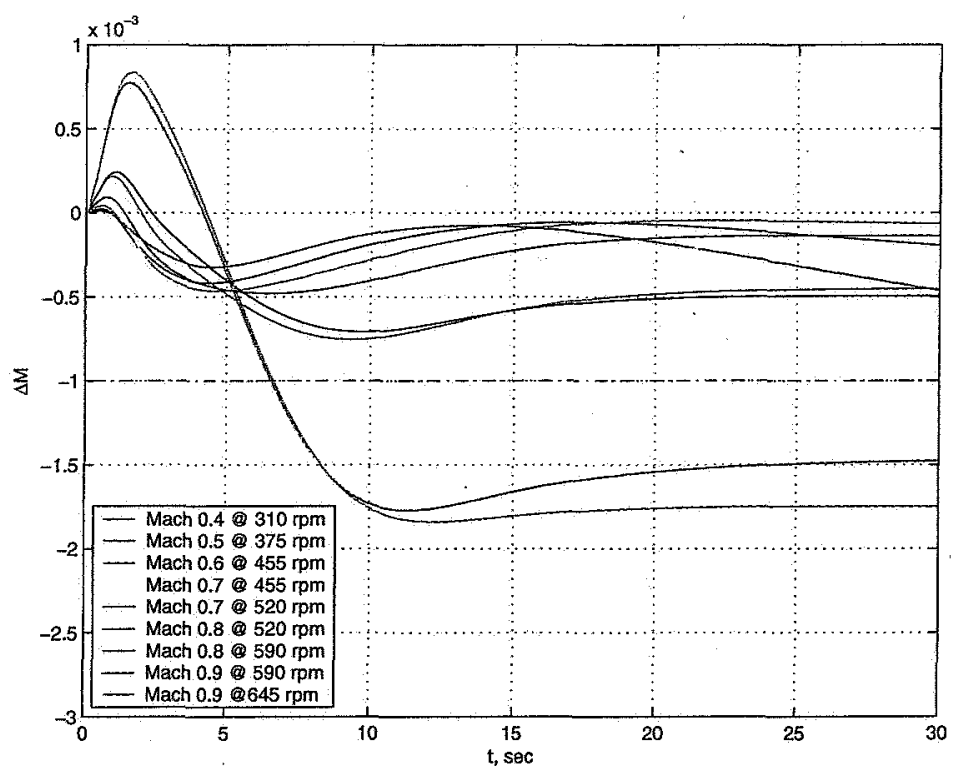

Fig. 13 - Mach Number Nonlinear Perturbation Response

To demonstrate the effectiveness of the model predictive control scheme for $\gamma=1$ over the subsonic operating envelope, we perform a control simulation for all Mach numbers from 0.4 to 0.9 . The results are plotted in Fig. 13 . As can be seen, the predictive control scheme for $\gamma=1$ is highly effective for all Mach numbers up to 0.8. The Mach number perturbation is well within the tolerance of \pm 0.001 . However, at a Mach number of 0.9 , there is a rapid increase in the Mach number perturbation that exceeds the tolerance. However, the largest perturbation is about -0.0018 which may be acceptable. The sudden change in the Mach number perturbation at a Mach number of 0.9 is directly attributed to the well-known phenomenon of transonic flow where many linear perturbation theories are broken down near a Mach number of unity. 
It should be noted that in the above model predictive control simulation, we assume a perfect knowledge of the drag coefficient of the test model. In reality, this information is generally not necessarily known in advance. Therefore, in order to implement a predictive control, a parameter estimation process must be incorporated to provide an estimation of the disturbance. In general, the accuracy of a predictive control is predicated upon the goodness of the estimation of the disturbance. Any significant deviation from the true value of the estimated parameter of the disturbance will likely cause the Mach number to exceed the tolerance band. Since the total pressure disturbance is a function of the drag force experienced on the test model, it may be readily estimated from a typical wind tunnel measurement of the three aerodynamic force components, namely; axial force coefficient $C_{A}$, side force coefficient $C_{S}$, and normal force coefficient $C_{N}$. These force components can be resolved in a direction parallel to the test section longitudinal axis using the Eulerian angles of pitch $\psi$, yaw $\phi$, and roll $\varphi$ from which the drag coefficient $C_{D}$ can be estimated according to

$$
C_{D}=C_{A} \cos \psi \cos \phi+C_{S}(\cos \psi \sin \phi \cos \varphi-\sin \psi \sin \varphi)+C_{N}(\cos \psi \sin \phi \sin \varphi+\sin \psi \cos \varphi)
$$

During a pitch polar, the aerodynamic forces generally vary as functions of the pitch angle as well as the Mach number and the Reynolds number. A database of the aerodynamic forces can be established as a table lookup process for various combinations of the Mach number, the Reynolds number, and the pitch angle. In lieu of the lookup table, the aerodynamic force relationships can also be established via an adaptive parameter estimation process such as a recursive least-square or a neural network algorithm which provides a functional approximation of the aerodynamic force dependency. Using the knowledge of the time response of the model support system, a trajectory of the total pressure disturbance parameter $w(t)$ can then be predicted for a given pitch polar. The trajectory of the total pressure disturbance is then used to compute the corrective optimal control inputs for the model predictive control scheme. Using the model predictive results of the optimal control, the compressor control is switched to an open-loop mode and begins its actuation simultaneously with the model support system. At the end of the feedforward mode, the compressor control is then switched back to a feedback mode. The model predictive Mach number control scheme thus potentially offers a significant advantage over the conventional feedback approach which has been known to be unable to regulate the Mach number during a continuous pitch polar in the NASA Ames 11-Foot Transonic Wind Tunnel

\section{Conclusion}

This paper describes a new model predictive optimal control method for the NASA Ames 11-Foot Transonic Wind Tunnel. The wind tunnel is modeled by the 1-D unsteady Euler equations whose boundary conditions prescribe a controlling action to the wind tunnel by an empirical compressor performance model. Furthermore, the compressor performance in turn is controlled by the drive motors and the inlet guide vane system whose dynamics are prescribed by a set of ordinary differential equations. In effect, the Euler equations become coupled to the ordinary differential equations via the boundary conditions. This wind tunnel math model has demonstrated an excellent agreement with test data. An adjoint method is used to establish necessary conditions of optimality of this type of systems. The theoretical results are then used to develop a model predictive optimal control that can be computed by solving the Riccati matrix equations. By shifting the control perturbation inputs ahead by a time delay between the test section and the compressor inlet as computed by the math model, the model predictive control is able to maintain the test section Mach number during a continuous pitch motion as demonstrated by a simulation study. The results of this study shows that the model predictive Mach number control potentially offers a much better disturbance rejection than a Mach number feedback control.

\section{References}

1. Soeterboek, R., Pels, A., Verbruggen, H., and van Langen, G., "A Predictive Controller for Mach number in a Wind Tunnel", IEEE Control Systems, 1995, pp 63-72.Soeterboek, R., et al, "A Predictive Controller for Mach number in a Wind Tunnel", IEEE Control Systems, 1995, pp 63-72.

2. Saunder, G., "UPWT Modernization Final Design Test Section Model Revision for the 11-Ft Tunnel Simulation", Contract No. NAS̄ $-1 \overline{3} 0 \overline{3} 2$, Sverdrup Technology, $1 \overline{9} \overline{9} \overline{1}$.

3. Shapiro, A., The Dynamics and Thermodynamics of Compressible Fluid Flow, Vol. 1, New York, John Wiley \& Sons, 1953.

4. Hirsh, C., Numerical Computation of Internal and External Flows, Vol. 1, John Wiley \& Sons, Brussels, 1991. 
5. Nguyen, N. and Ardema, M., "Optimal Control of Flow Recirculation in a Wind Tunnel", AIAA 2004-4759, August 2004.

6. Raymond, J. and Zidani, H., "Pontryagin's Principle for State-Constrained Control Problems Governed by Parabolic Equations with Unbounded Controls", SIAM Journal of Control Optimization, Vol. 36, No. 3, 1998 , pp. 1853-1879.

7. Troltzsch, F., "On the Lagrange-Newton-SQP Method for the Optimal Control of Semilinear Parabolic Equations", SLAM Journal of Control Optimization, Vol. 38, No. 1, 1999, 294-312.

8. Kazemi, M., “A Gradient Technique for an Optimal Control Problem Govemed by a System of Nonlinear First Order Partial Differential Equations", Journal of Australian Mathematical Society, Serial B 36 (1994), 261-273.

9. Fursikov, A., Gunzburger, M., and Hou, L., "Boundary Value Problems and Optimal Boundary Control for the Navier-Stokes System: the Two-Dimensional Case", SIAM Journal of Control Optimization, Vol. 36, No. 3, 1998, pp. 852-894.

10. Jameson, A., Pierce, N., and Martinelli, L., "Optimum Aerodynamic Design Using the Navier-Stokes Equations", Theoretical Computational Fluid Dynamics, Vol. 10, 1998, pp. 213-237.

11. Nadarajah, S., Jameson, A., and Alonso, J., "Sonic Boom Reduction Using an Adjoint Method for Wing-Body Configurations in Supersonic Flow", AIAA 2002-5547, 9th AIAA/ISSMO Symposium on Multidisciplinary Analysis and Optimization Conference, Atlanta, GA, September 4-6, 2002. 